\title{
Détection des cannelures du tronc de la vigne par indexage en vert (greffe-bouture herbacée)
}

\author{
P Bass 1, A Dumont 2 , C Greif $1^{\star}$, B Walter 1 \\ 1 INRA, station de recherches Vigne et Vin, laboratoire de Pathologie végétale, BP 507, F68021 Colmar cedex; \\ 2 Mumm et Perrier Jouët, Vignobles et Recherches, BP 186, F51206 Épernay cedex, France
}

(Reçu le 7 janvier 1993; accepté le 19 mars 1993)

\begin{abstract}
Résumé - L'utilisation de la greffe-bouture herbacée pour le dépistage des cannelures du tronc de la vigne par indexage a nécessité la mise au point des conditions d'extériorisation des symptômes appropriées à la détection précoce de ces syndromes. Les symptômes, observés sur des coupes transversales du bois de l'indicateur juste en dessous du point de greffe, apparaissent, dans le cas du Kober stem grooving (KSG), 6 à 8 mois après greffage sur Kober 5BB. Le diagnostic du Rupestris stem pitting (RSP) sur Rupestris du Lot demande en revanche plus d'1 an pour un développement équivalent des symptômes. Le porte-greffe 161-49C, utilisé comme indicateur du RSP, répond plus rapidement que Rupestris du Lot. Malgré une certaine hétérogénéité de réaction, liée au degré d'aoûtement de la tige d'indicateur, il permet généralement d'établir un diagnostic 8 mois après le greffage. L'application de cette méthode à la sélection sanitaire, ainsi qu'à l'étude étiologique des cannelures, est discutée.
\end{abstract}

vigne / indexage en vert (greffe-bouture herbacée) / cannelures du tronc / Kober stem groowing / Rupestris stem pitting / sélection sanitaire

Summary - Use of green-graft indexing for early detection of grapevine stem grooving / pitting diseases. Controlled growth conditions (temperature, light, raising) were developed for early and reliable symptom expression by green-graft indexing of 2 syndromes of the grapevine rugose wood complex: Kober stem grooving (KSG) and Rupestris stem pitting (RSP). Because of the young plant material, symptoms could not be revealed after peeling of the indicator trunk and so were observed by light microscopy on transverse sections of the indicator stem just below the graft point. After 6 to 8 months KSG symptoms on Kober 5BB were more marked than RSP symptoms on Rupestris $d u$ Lot and were obvious enough to establish the presence of the disease, whereas RSP diagnosis required more than a year. Despite these differences in symptom development the cytopathological effects on the indicator wood appeared very similar between the 2 syndromes. The rootstock hybrid 161-49 C used as RSP indicator showed symptoms sooner than Rupestris du Lot and, in spite of a greater variability in the responses linked to the extent of lignification of the indicator stem, generally gave a conclusive result after 8 months. The results also showed that the presence of a Vitis vinifera in the graft combination is indispensable for the expression of the KSG and RSP symptoms on the indicators. The application of green-graft indexing for KSG and RSP to sanitary selection as well as to the etiological study of these diseases has been discussed.

grapevine / green-graft indexing / rugose wood / Kober stem grooving / Rupestris stem pitting / sanitary selection

\section{INTRODUCTION}

Les cannelures du tronc de la vigne sont des maladies de type viral longtemps décrites sous le terme générique de legno riccio (Graniti, 1964 ; Graniti et Martelli, 1970), qui en fait regroupait au moins 4 syndromes que l'on distin- gue actuellement par les réactions qu'ils provoquent sur des variétés indicatrices différentes (Savino et al, 1989) : le Rupestris stem pitting (RSP, ou cannelures sur Rupestris du Lot; Bovey et al, 1980 ; Goheen, 1988), le Kober stem grooving (KSG, ou cannelures sur Kober $5 B B$; Legin et al, 1979), le LN 33 stem grooving (Garau et al, 1989) et l'écorce liégeuse

* Correspondance et tirés à part 
(corky bark; Hewitt et al, 1962). Le terme de cannelures du tronc se rapporte usuellement aux 2 premiers syndromes. Leur incidence économique est plus ou moins importante (Garau et al, 1985), mais le fait qu'elles soient répandues dans le monde entier et que certains assemblages puissent être à l'origine d'effets graves sur la vigueur du porte-greffe (Lehoczky, 1972; Savino et al, 1985) accroît la nécessité de développer une méthode de diagnostic rapide et fiable.

L'indexage du RSP et du KSG par greffage traditionnel de bois aoûtés (Graniti et Martelli, 1965 ; Legin, 1972 ; Hewitt, 1975), encore communément utilisé, demande une durée de notations assez longue, 2 ans en moyenne, et un espace important pour l'élevage des plants greffés en pépinière. De plus, la période de greffage est limitée dans l'année (de février à avril en conditions septentrionales). L'indexage en vert, dont le principe a depuis longtemps été appliqué à la vigne pour la détection de l'écorce liégeuse (Hévin et al, 1973; Mink et Parsons, 1977), se présentait comme une méthode de diagnostic plus rapide, mais s'effectuait dans ce cas sur porte-greffes racinés. La greffe-bouture herbacée, utilisée pour le dépistage des maladies de type viral de la vigne (Walter et al, 1990), a permis de réduire la durée du diagnostic de ces maladies à quelques mois, ainsi que l'espace nécessaire aux tests, mais n'a pu dans un premier temps être appliquée aux cannelures du tronc.

Nous avons dû mettre au point les conditions nécessaires à l'extériorisation reproductible des symptômes de cannelures sur leurs indicateurs et trouver une méthode de détection des bois infectés adaptée à un matériel très jeune. Nous présentons dans ce travail l'éventail des symptômes observés avec les différents indicateurs que nous utilisons pour l'indexage du KSG et du RSP.

\section{MATÉRIEL ET MÉTHODES}

\section{Matériel végétal}

Les variétés de porte-greffe utilisées comme indicateurs des cannelures sont le Vitis rupestris Scheele St George, ou Rupestris du Lot, et les hybrides $V$ berlandieri $\times \vee$ riparia Michaux Kober 5BB, SO4 et 161 $49 \mathrm{C}$. Les témoins malades sont des clones de $V$ vinifera : Servant A94 pour le KSG (Legin et al, 1979) et
Pinot Noir 14 pour le RSP. Les témoins sains sont des clones de $V$ vinifera traités par thermothérapie et/ou greffe d'apex (Bass et Legin, 1981).

\section{Obtention du matériel herbacé}

Les indicateurs sont élevés en serre, sur pain de laine de roche, à une densité de 20 pieds au $\mathrm{m}^{2}$. Leur nutrition est assurée par un réseau de goutteurs distribuant un engrais liquide adapté (Huglin et Julliard, 1964). La température d'ambiance est d'un minimum de $17^{\circ} \mathrm{C}$ la nuit et doit atteindre au moins $24^{\circ} \mathrm{C}$ le jour.

Les échantillons à analyser sont mis à forcer, sous forme de boutures talonnées à un œil, en pots de 7,5 I dans un mélange de sable/perlite (60/40). Ces pots contenant au maximum 10 boutures sont installés pendant 1 mois en serre chaude $\left(28^{\circ} \mathrm{C}\right.$ constant). Ils sont ensuite transférés en serre, dans des conditions d'élevage identiques à celles des indicateurs.

\section{Greffage}

Les boutures d'indicateurs et les greffons (échantillons à indexer) sont assemblés à l'aide d'une machine à greffer Greffenvert (brevet Mumm et Perrier Jouët FR A 860 1117) qui réalise une greffe en fente pleine. Le maintien des éléments de la greffe est assuré par une mini-épingle à linge. Des groupes de 6 assemblages de l'échantillon avec l'indicateur requis sont piqués sur un bloc de laine de roche trempé au préalable dans la solution nutritive additionnée de $5 \mathrm{mg} / \mathrm{hl}$ d'acide naphtalène acétique, puis sont introduits dans des minienceintes hermétiquement fermées. La phase de soudure-rhizogenèse des plants greffés est réalisée dans les conditions décrites par Martin et al (1987).

\section{Cas particulier des doubles-greffes}

Si l'échantillon à tester est une variété de porte-greffe (espèce américaine, hybride d'espèces américaines), l'assemblage suivant est réalisé : un $V$ vinifera sain est greffé sur l'échantillon, lui-même greffé sur l'indicateur.

\section{Conditionnement à l'extériorisation des symptômes}

Au moment de la sortie des mini-enceintes, chaque porte-greffe est éborgné, seul l'échantillon est conservé poussant. Dans le cas des doubles-greffes, l'échantillon est lui aussi éborgné et le $V$ vinifera seul est conservé poussant. Les groupes sont installés pendant 2 à 3 sem dans une serre dite de sevrage, pour favoriser le démarrage de la pousse apicale de l'assemblage. À l'issue de cette période, les groupes sont transférés dans une serre équipée de gouttières, per- 
mettant l'arrosage par subirrigation. Les tiges poussantes sont palissées. Les conditions d'élevage sont les mêmes que celles décrites pour les porte-greffes indicateurs.

\section{Notations}

Le diagnostic se fait par coupe transversale de l'indicateur, juste en dessous du point de greffe, dès qu'une majorité de groupes a atteint un aoûtement suffisant et à condition qu'au moins 3 greffés-soudés par groupe aient survécu. La coupe, d'environ 80 à $150 \mu \mathrm{m}$, est réalisée à main levée, avec une lame de rasoir. L'examen de la coupe est effectué dans l'eau, entre lame et lamelle, à la loupe binoculaire. Dans tous les cas, l'établissement du diagnostic nécessite la présence de témoins sains et malades produits le même jour que les groupes à tester.

\section{RÉSULTATS}

\section{Mise au point de la méthode de détection}

Les premiers essais d'indexage par greffebouture herbacée, concernant le Kober stem grooving, ont montré qu'il fallait attendre 6 à 8 mois après le greffage, selon la période de l'année, pour obtenir une majorité de plants aoûtés. Les cannelures étant décrites traditionnellement comme des sillons longitudinaux apparaissant à la surface du bois de l'indicateur après décortication (Bovey et al, 1980), nous avons tout d'abord cherché à les retrouver sur ce matériel beaucoup plus jeune. À cette fin, nous avons choisi une technique d'élevage des plants en gouttière (fig 1) favorable à la croissance en épaisseur de l'indicateur. Mais en dépit d'un aoûtement complet, l'écorce s'est révélée trop mince et la décortication trop difficile pour permettre un jugement direct. Cependant, certains groupes d'indexage présentaient, outre une couleur anormalement foncée par rapport au témoin sain et une nette réduction du système racinaire (fig 2), des stries visibles juste en dessous du point de greffe. Afin de vérifier la fiabilité d'un diagnostic basé uniquement sur ces observations externes, un essai avec des clones d'état sanitaire connu a été réalisé. II s'est avéré que l'aspect externe du bois était insuffisant pour délivrer un diagnostic sûr.

Des observations de coupes effectuées dans la tige du porte-greffe ont permis de mettre en

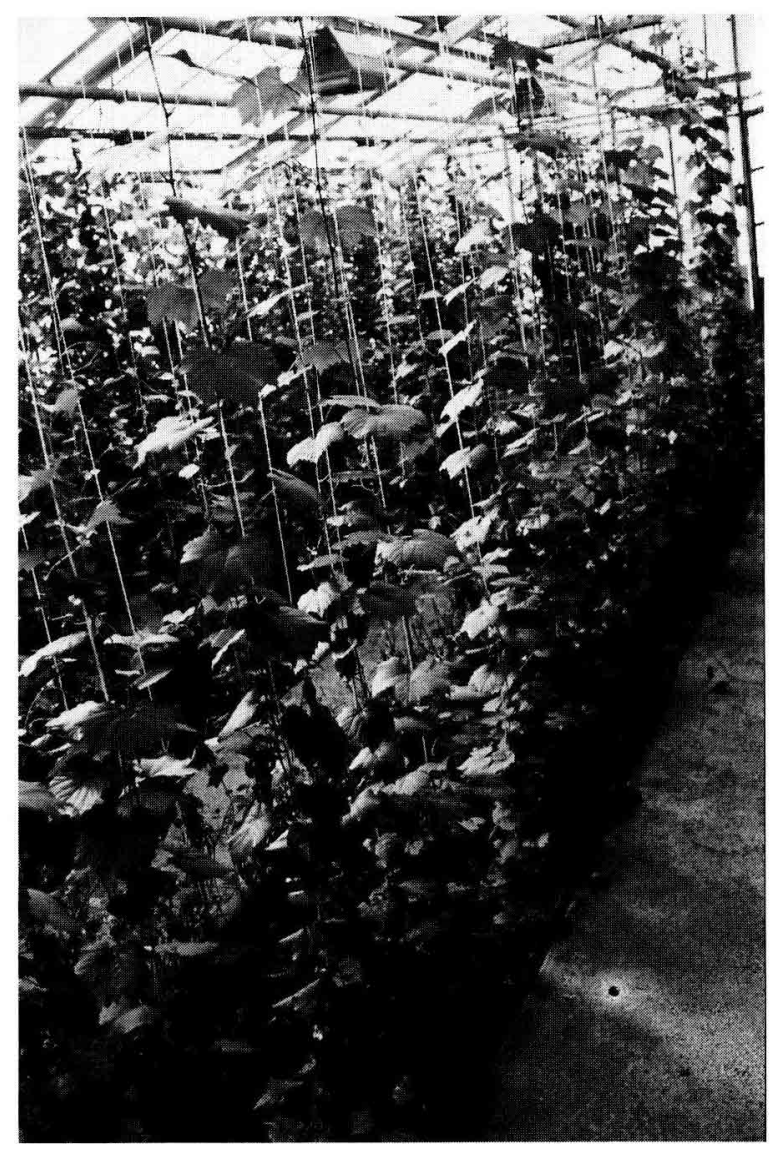

Fig 1. Élevage des plants greffés-soudés en gouttière pour la détection des cannelures du tronc par indexage en vert.

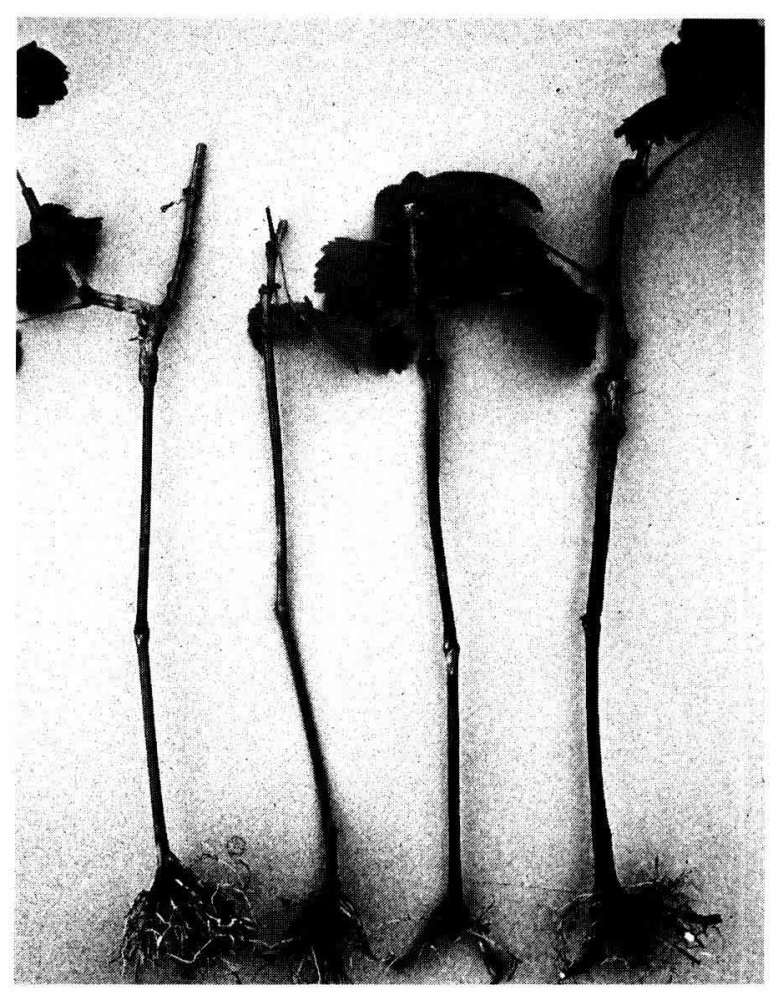

Fig 2. Effet du KSG sur le développement du système racinaire de l'indicateur. À gauche, Kober 5BB greffé avec un témoin sain (Chardonnay guéri par thermothérapie); à droite, 3 échantillons d'un même groupe d'indexage (Servant A94/ Kober 5BB). À noter, en plus de la réduction du nombre de racines, les différents degrés d'aoûtement des tiges d'indicateur. 
évidence des dérangements tissulaires majeurs, visibles chez la totalité des individus présentant des stries externes, mais aussi chez d'autres individus ne montrant aucun symptôme apparent, si les coupes étaient pratiquées juste en dessous du point de greffe (fig 3 ).

L'application de cette méthode à la détection du Rupestris stem pitting a révélé un retard beaucoup plus prononcé de l'aoûtement du porte-greffe, nécessitant d'attendre la deuxième année de végétation (comme en indexage traditionnel) pour obtenir une majorité de bois aoûtés dans les groupes d'indexage et des symptômes de gravité équivalente à ce qui avait été observé sur les coupes de Kober 5BB (fig 4).

L'examen des coupes a montré que les 2 types de cannelures, bien que s'exprimant sur des variétés différentes de porte-greffe, induisent des phénomènes comparables au stade final d'expression des symptômes. Alors que les coupes concernant des individus sains montrent un contour régulier (fig 3 ), les coupes correspondant à des individus malades présentent un contour tout à fait irrégulier et une désorganisation sévère des différents tissus et de leur agencement (figs 3 et 4). Cette désorganisation se traduit par :

- la présence d'une épaisse couche brunâtre au niveau du cortex, constituée d'anciens tissus nécrosés ;

- la présence de pointes nécrotiques au sommet des rayons médullaires pouvant s'étendre sur toute l'épaisseur de la zone libéro-ligneuse ;

- l'accolement de certains rayons médullaires ;

- l'apparition précoce de cellules nécrosées à la périphérie de la moelle, au niveau des pôles ligneux ;

- la diminution apparente en taille et en nombre des vaisseaux du bois ;

- la très forte réduction de la taille des massifs de fibres péricycliques (voire leur disparition).

D'autre points communs ont été observés par ailleurs :

- le diamètre sous le point de greffe des indicateurs des groupes malades est toujours supérieur à celui des témoins sains du même âge ;

- la présence de tiges partiellement aoûtées dans un groupe d'indexage est un signe précurseur de la maladie ;

- dans les 2 cas, la maladie se traduit par une réduction du système racinaire.

\section{Utilisation d'autres variétés indicatrices des cannelures}

II arrive que certains groupes dindexage sur Kober 5BB ne puissent être jugés quand les greffons se développent mal et finissent par mourir, révélant le phénomène dit «d'incompatibilité» au greffage (Durquety et al, 1973 ; Legin et Walter, 1986). L'indicateur reste alors vert ou mal aoûté et l'examen des coupes après 6 à 8 mois ne donne aucun résultat interprétable. L'indexage de ces échantillons peut être effectué sur une autre variété de porte-greffe, le $\mathrm{SO}_{4}$ (Legin et Walter, 1986), qui exprime des symptômes de KSG semblables à ceux observés sur Kober 5BB (fig 5).

Pour le RSP, l'expression tardive des symptômes sur l'indicateur Rupestris du Lot nous a amenés à rechercher un indicateur susceptible de répondre plus précocement. Parmi les différentes variétés déjà testées pour le RSP en indexage traditionnel, le 161-49 C s'était révélé être un bon indicateur (Valat et al, 1981; R Legin, communication personnelle). Huit mois après le greffage en vert sur 161-49 C, les groupes testés étaient en grande majorité suffisamment aoûtés et des dérangements au niveau des tissus étaient déjà visibles sur les coupes (fig 6). Cependant, au fur et à mesure des tests effectués sur 161-49 C, une certaine hétérogénéité de réponse est apparue, non seulement entre les groupes d'indexage, mais aussi à l'intérieur d'un même groupe. Les différentes réponses obtenues ont pu être classées par ordre croissant, presque "chronologique», de détérioration des tissus libériens et ligneux:

- symptômes de type vert (fig 7a) ou mal aoûté (fig $7 \mathrm{~b}$ ) : la partie libérienne des rayons médullaires est élargie et reste totalement verte ; le liber revêt un aspect anormal en arches ; la nécrose des tissus du cortex est très irrégulièrement répartie ; le tissu ligneux ne semble pas affecté ;

- symptômes de type régulier ou dentelé (fig 7c, d) : la couche de tissus nécrosés du cortex est plus régulière et semble suivre une progression centripète ; la boucle des arches de cellules libériennes est située maintenant dans la partie brune du cortex, au milieu des tissus nécrosés ; le phellogène est déformé par de faibles incursions de tissus nécrosés dans la zone libérienne des rayons médullaires (aspect dentelé, voir aussi fig 6); l'anneau ligneux est progressivement atteint (nécroses dans le xylème, obturation des vaisseaux) ; 

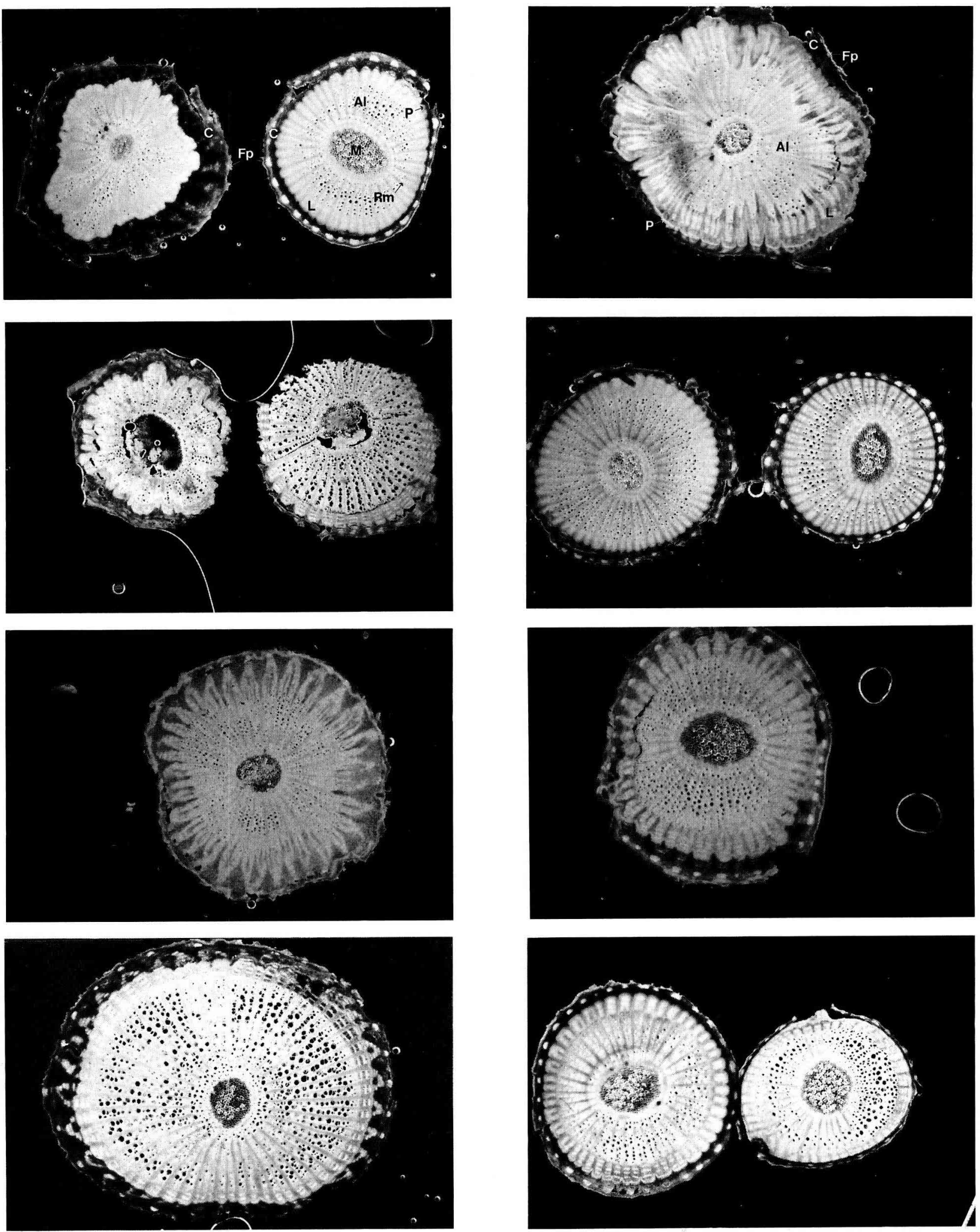

Figs 3 à 7. 3. Symptômes de KSG sur Kober 5BB après 8 mois. À gauche, coupe de Kober $5 B B$ greffé avec le témoin malade (Servant A94); à droite, coupe de Kober 5BB greffé avec un témoin sain (Klevener TG64). Al : anneau ligneux; $\mathrm{C}: \mathrm{cortex}, \mathrm{Fp}$ : fibres péricycliques, $L$ : liber, $M$ : moelle, $P$ : phellogène (assise génératrice), $R m$ : rayon médullaire. 4. Symptômes de RSP sur Rupestris du Lot en deuxième année de végétation. Coupe de Rupestris du Lot greffé avec un témoin malade (Pinot noir 14). 5. Symptômes de KSG sur $\mathrm{SO}_{4}$ après 8 mois. A gauche, coupe de $\mathrm{SO}_{4}$ greffé avec le Servant A94; à droite, coupe de $\mathrm{SO}_{4}$ greffé avec un témoin sain (Klevener TG64). 6. Symptômes de RSP sur 161-49 C après 8 mois. À gauche, coupe de 161-49C greffé avec un clone malade de Perle de Csaba; à droite, coupe de 161-49C greffé avec un clone de Perle de Csaba issu du même plant-mère guéri par thermothérapie et greffe d'apex. 7. Différents types de symptômes de RSP observés sur 161-49C après 8 mois. Coupes de 161-49C greffé avec différents échantillons de chardonnay. a. Symptômes de type vert; $\mathbf{b}$. Symptômes de type mal aoûté; $\boldsymbol{c}$ et $\mathbf{d}$. Symptômes de type régulier ou dentelé; $\boldsymbol{d}$. À droite : coupe de 161-49C sain non greffé. 
- symptômes de type sévère : le liber est gravement déformé ; le brunissement atteint des zones plus profondes du bois; les symptômes sont semblables à ceux observés sur Rupestris du Lot.

Les 2 premiers types de symptômes se retrouvent très souvent au sein d'un même groupe d'indexage, alors que le symptôme sévère est rare sur des greffes de 8 mois (il faut en général attendre plus de 1 an, comme pour $V$ rupestris). La coexistence des 2 types de symptômes, "vert» et «dentelé», peut s'expliquer par la différence d'âge physiologique des boutures d'indicateur prélevées le jour du greffage : sur une tige herbacée de $2 \mathrm{~m}$, les boutures du haut ont environ 6 sem de moins que celles du bas, leur phase d'aoûtement est donc quelque peu retardée. Ces phénomènes ne s'observent par sur Kober $5 \mathrm{BB}$ où les symptômes sont très sévères dès 6 à 8 mois, même si l'indicateur n'est pas totalement aoûté, ni sur Rupestris du Lot, pour lequel aucun symptôme n'est apparent après 8 mois.

\section{Implication de $\mathrm{V}$ vinifera dans l'expression des symptômes de cannelures}

Lorsque le test d'indexage concerne une variété de porte-greffe, la présence d'un $V$ vinifera sain pour les cannelures - dans la combinaison de greffe est indispensable pour le développement des symptômes de cannelures sur l'indicateur. Un échec de la greffe entre le $V$ vinifera et un échantillon malade empêche l'extériorisation des cannelures sur l'indicateur, même quand le $V$ vinifera survit pendant un certain temps sans pousser. Par ailleurs, si le porte-greffe indicateur, accidentellement mal éborgné, émet un

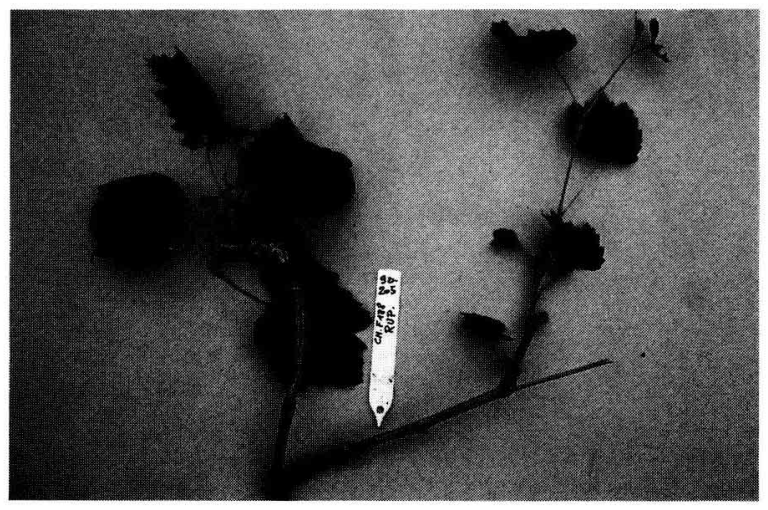

rejet, celui-ci, qui n'est pas en contact direct avec le $V$ vinifera, ne montre aucun symptôme de cannelures (fig $8 a$ et $b$ ).

\section{DISCUSSION}

Dans les conditions classiques d'indexage de la vigne, un des critères pour une bonne expression des symptômes de cannelures du tronc est la forte croissance du plant greffé, et notamment du tronc de l'indicateur (V Savino, communication personnelle). Nous avons été confrontés à ce type de problème lors de l'application de la greffe-bouture herbacée au diagnostic des cannelures par indexage, car l'épaisseur et le degré d'aoûtement de la tige de l'indicateur sont des facteurs limitants pour établir le diagnostic. En effet, même si la transmission de la maladie à l'indicateur entraîne un grossissement de sa tige par rapport à un témoin sain, des résultats clairs et reproductibles ne sont obtenus qu'avec des tiges d'indicateur d'au moins $4 \mathrm{~mm}$ de diamètre. C'est pourquoi nous élevons les groupes d'indexage en gouttière, de manière à favoriser la croissance en épaisseur du porte-greffe. Par ailleurs, chez l'indicateur, le développement de la maladie et la maturation du bois se font en parallèle, ce qui occasionne un retard à l'aoûtement, très marqué chez le Rupestris du Lot, un peu moins chez les autres indicateurs, et qui détermine le temps minimum nécessaire pour l'établissement du diagnostic selon l'indicateur utilisé. II apparaît ainsi que 6 à 8 mois sont suffisants pour le KSG jugé sur Kober $5 \mathrm{BB}$ et $\mathrm{SO}_{4}$, qu'au moins 12 à 14 mois sont nécessaires pour le RSP jugé sur Rupestris du Lot, mais que 8 mois suffisent pour une grande partie des tests RSP jugés sur 161-49 C.

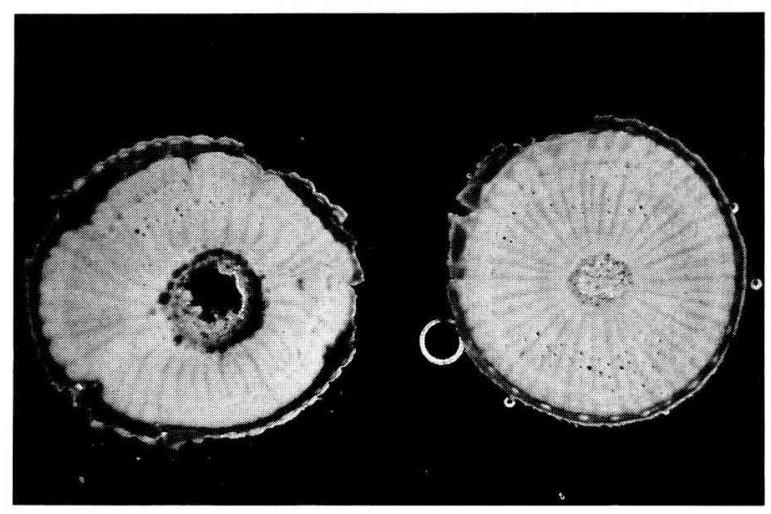

Fig 8. Aspect sain d'un rejet développé sur une bouture non éborgnée de Rupestris du Lot greffée à un chardonnay atteint de RSP. a. Vue d'ensemble du plant greffé; b. Comparaison d'une coupe de l'indicateur sous le point de greffe (à gauche) et d'une coupe du rejet (à droite). 
Dans ce dernier cas, nous avons pu remarquer l'incidence de l'âge physiologique de la bouture de l'indicateur utilisée lors du greffage sur le niveau d'expression des symptômes au moment du diagnostic. L'utilisation d'un matériel végétal plus homogène nous permettrait d'estomper ce phénomène, mais nécessiterait une production de plantes beaucoup plus importante, qu'il n'est pas concevable d'appliquer à de grandes séries d'indexage pour des raisons pratiques. L'absence totale de dérangements sur Kober 5BB ou 161-49 C lorsque le greffon est un $\checkmark$ vinifera guéri des cannelures par thermothérapie et/ou greffe d'apex (figs 2 et 7 ) a confirmé la validité de nos tests, montrant que les dérangements tissulaires observés ne pouvaient être des artefacts liés aux nouvelles conditions d'indexage. D'autre part, dans ces conditions contrôlées d'indexage nous avons pu retrouver de façon très précoce (moins de 1 an) l'expression de l'ensemble des phénomènes décrits auparavant en indexage traditionnel, comme certains dérangements tissulaires (Garau et al, 1974; Boubals et al, 1976 ; Kriel et al, 1980), l'effet sur le système racinaire du porte-greffe (Legin, 1972) ou les réponses obtenues avec d'autres indicateurs, $\mathrm{SO}_{4}$ (Legin et al, 1979) et 161-49 C (Valat et al, 1981). Mais, dans le cas présent, l'utilisation de matériel végétal vert a mis au jour des observations nouvelles, notamment sur la progression de la maladie au niveau des tissus, qu'il conviendrait d'affiner par une étude histologique de séries de coupes fines, effectuées selon une cinétique régulière pendant toute la durée du test.

D'autres observations soulignent un aspect jusqu'ici inabordé qui est celui des interactions entre $V$ vinifera et les variétés de porte-greffe indicatrices des cannelures pour l'expression des symptômes sous le point de greffe : la nécessité d'inclure un $V$ vinifera dans la combinaison de greffe quand une variété de porte-greffe (espèce américaine ou hybride d'espèces américaines) est indexée, alors qu'il n'en est aucun besoin pour des hybrides interspécifiques ayant un parent $V$ vinifera (eg LN33, 41B; Engelbrecht et al, 1991; R Legin, communication personnelle), et l'absence de symptômes sur le rejet d'un indicateur, qui exprime nettement la maladie sous le point de greffe. Des études supplémentaires seront nécessaires pour essayer de définir les bases de ces interactions. Quoi qu'il en soit, il est important de tenir compte de ces indications pour la mise en place d'un protocole d'indexage des cannelures.
La possibilité d'utiliser la méthode de greffebouture herbacée pour la détection des cannelures du tronc de la vigne est évidemment d'un grand intérêt pour la sélection sanitaire. Cependant, dans cette optique il faut encore prendre en compte les caractéristiques propres aux 2 types de cannelures. D'une part, les fréquences des deux syndromes sont différentes : le RSP est retrouvé dans un nombre très important de clones commerciaux de $V$ vinifera et de portegreffes (Goheen, 1988; Garau et al, 1989), alors que le KSG est beaucoup moins souvent détecté et généralement associé à l'enroulement. D'autre part, la guérison par thermothérapie et greffe d'apex du KSG est obtenue sans trop de difficulté, en relation étroite avec l'enroulement (Legin et al, 1979 ; Bass et Legin, 1981), alors qu'elle tient beaucoup plus du hasard pour le RSP (Habili et al, 1992). Si la sélection sanitaire peut donc s'entrevoir dans le cas du KSG, le problème n'est pas encore résolu pour le RSP. Nous analysons actuellement un grand nombre de clones de vigne issus de thermothérapie et culture in vitro pour avoir une meilleure idée des taux de réussite de guérison du RSP et évaluer les performances culturales des plants guéris.

Enfin, il importe de bien distinguer les 2 maladies et de profiter de la rapidité offerte par la méthode de greffe-bouture herbacée pour aller plus loin dans leur caractérisation et dans l'étude de leur étiologie, notamment en ce qui concerne l'existence probable d'isolats plus ou moins virulents de l'agent responsable du RSP (Garau et al, 1991) et l'implication précise du grapevine virus $A$ qui a été associé, de manière inconstante, à la maladie des cannelures (Gugerli et al, 1991).

\section{REMERCIEMENTS}

Les auteurs tiennent à remercier M R Legin pour son concours à l'adaptation de la méthode, de même que Mlle $L$ Carde, le professeur JC Audran pour la description des symptômes sur coupes et MM JM Barillère et A Collas pour leur soutien durant tout ce travail.

\section{RÉFÉRENCES}

Bass P, Legin R (1981) Thermothérapie et multiplication in vitro d'apex de vigne. Application à la séparation ou l'élimination de diverses maladies de type viral et à l'évaluation des dégâts. $C R$ Séances Acad Agric Fr 67, 922-933 
Boubals D, Pistre R, Giraud G, Roche J (1976) Distribution du symptôme des cannelures du tronc (bois strié) dans des vignes atteintes de court-noué et des vignes infectées par l'enroulement. Prog Agric Vitic 93, 634-642

Bovey R, Gärtel W, Hewitt WB, Martelli GP, Vuittenez A (1980) Maladies à virus et affections similaires de la vigne. Payot, Lausanne, $181 \mathrm{p}$

Durquety PM, Fallot J, Ruchaud C, Benassac JP, Dauty R (1973) Le clone et ses réactions au greffage. Existence, dans un cépage population, de clones présentant divers degrés de compatibilité avec certains porte-greffes. Prog Agric Vitic 90, $122-129$ et $171-178$

Engelbrecht DJ, Kasdorf GGF, Maré FA (1991) Field spread of stem-grooving diseases in South African grapevines. Phytophylactica 23, 239-240

Garau R, Prota U, Servazzi O (1974) Osservazioni istologiche su viti portinnesto con sintomi riferibili al "legno riccio». Phytopathol Mediterr 13, 64-70

Garau R, Cugusi M, Dore M, Prota U (1985) Investigations on the yields of "Monica» and «Italia» vines affected by legno riccio (stem pitting). In: Proc 8 th ICVG meeting. Bari, Italy, 1984. Phytopathol Mediterr 24, 64-67

Garau R, Prota U, Cugusi M (1989) Investigations on wood disorders (stem pitting and/or stem grooving) of grapevine in Sardinia. In: Proc 9th ICVG Meeting Kiryat Anavim, Israel, 1987, 135-141

Garau R, Prota VA, Prota U (1991) Distribution of Kober stem grooving and Rupestris stem pitting of grapevine in symptomless cv Torbato scions. In: Proc 10th ICVG Meeting. Volos, Greece, 1990, 175-181

Goheen AC (1988) Rupestris stem pitting. In: Compendium of grape diseases (Pearson RC, Goheen $A C$, eds) APS Press, Saint-Paul, $93 p$

Graniti A (1964) Note syntomatologiche e istologiche sulle viti affette da «legno riccio». Phytopathol Mediterr 3, 19-25

Graniti A, Martelli GP (1965) Further investigations on legno riccio (rugose wood), a graft transmissible stem pitting of grapevine. Proc int conf on virus and vector on perennial hosts with special reference to Vitis. Davis, USA, 168-179

Graniti A, Martelli GP (1970) Legno riccio. In: Virus diseases of small fruits and grapevines: $A$ handbook (Frazier NW, ed) Univ California, Div Agric Sci, Berkeley, CA, 243-245

Gugerli P, Rosciglione B, Brugger JJ, Bonnard S, Ramel ME, Tremea F (1991) Further characterisation of grapevine leafroll disease. In: Proc 10th ICVG meeting. Volos, Greece, 1990, 59-60

Habili N, Krake LR, Barlass M, Rezaian (1992) Evaluation of biological indexing and dsRNA analysis in grapevine virus elimination. Ann Appl Biol 121, 277-283

Hévin MM, Leclair P, Rives M (1973) Green-grafting as a quick and secure method for graft-indexing vi- ruses in the grapevine. Riv Pathol Veg Ser IV, 9, 277-278

Hewitt WB (1975) Graft transmission of a grapevine wood pitting and a flat trunk disease. Plant Dis Rep 59, 845-848

Hewitt WB, Goheen AC, Raski DJ, Gooding GV Jr (1962) Studies on virus diseases of the grapevine in California. Vitis 3, 57-83

Huglin P, Julliard B (1964) Sur l'obtention de semis de vigne très vigoureux à mise à fruits rapide et ses répercussions sur l'amélioration génétique de la vigne. Ann Amélior Plantes 14, 229-244

Kriel GJ le R, Orffer CJ, Beukman EF (1980) Symptomatology and anatomy of stemgrooving (legno riccio) in the grapevine. S Afr J Enol Vitic 1, 85101

Legin R (1972) Expérimentation pour étudier l'effet des principales viroses sur la végétation et la production de la vigne. In: IVth meeting of the ICVG. Colmar, France, 1970. Ann Phytopathol (hors série), 49-57

Legin R, Walter B (1986) Étude de phénomènes d'incompatibilité au greffage chez la vigne. Prog Agric Vitic 103, 279-283

Legin R, Bass P, Vuittenez A (1979) Premiers résultats de guérison par thermothérapie et culture in vitro d'une maladie de type cannelure (legno riccio) produite par le greffage du cultivar Servant de Vitis vinifera sur le porte-greffe $V$ riparia $\times V$ berlandieri Kober 5BB. Comparaison avec diverses viroses de la Vigne. Phytopathol Mediterr 18, 207-210

Lehoczky J (1972) Destructive effect of legno riccio (rugose wood) on European grapevine varieties. In: IVth meeting of the ICVG. Colmar, France, 1970. Ann Phytopathol (hors série) 59-65

Martin C, Vernoy R, Carré $M$, Vesselle $G$, Collas A, Bougerey $C$ (1987) Vignes et techniques de culture in vitro. Quelques résultats d'une collaboration entre recherche publique et entreprise privée. Bull OIV 675/676, 447-458

Mink GI, Parsons JL (1977) Procedures for rapid detection of virus-like diseases of grapevine. Plant Dis Rep 61, 567-471

Savino V, Boscia D, Musci D, Martelli GP (1985) Effect of legno riccio (stem pitting) on "Italia" vines grafted on rootstocks of different origins. In: Proc 8th ICVG meeting, Bari, Italy, 1984. Phytopathol Mediterr 24, 68-72

Savino V, Boscia D, Martelli GP (1989) Rugose wood complex of grapevine: can grafting to Vitis indicators discriminate between diseases? In: Proc 9th ICVG Meeting. Kiryat Anavim, Israel, 1987, 91-94

Valat C, Grenan S, Bonnet A (1981) La maladie du bois strié de la vigne. Progr Agric Vitic 98, 151-156

Walter B, Bass P, Legin R, Martin C, Vernoy R, Collas A, Vesselle $G$ (1990) The use of green-grafting technique for detection of virus-like diseases of grapevine. J Phytopathol 128, 137-145 